REVISTA DE DERECHO UNED, NÚM. 11, 2012

\title{
TRADICIÓN ROMANÍSTICA Y PERSPECTIVAS DE REFORMA EN SEDE DE CLÁUSULA PENAL
}

\author{
ROMAN TRADITION AND PERSPECTIVES ON REFORM \\ ON PENALTY CLAUSE
}

\author{
ALEJANDRo VALiÑo \\ Universidad de Valencia
}

\begin{abstract}
Resumen: El autor analiza el tratamiento legal de la cláusula penal en el Derecho civil español, con especial atención a la regulación que ofrece el Código civil en los artículos 1152 a 1154, las diferentes funciones que la cláusula penal cumple y el distinto régimen presente en la Lex 518 del Fuero Nuevo de Navarra, lo cual evidencia el dualismo habido en el sistema jurídico español entre el Derecho civil común (representado por el Código civil) y el derecho foral o especial (representado por distintas compilaciones de ámbito regional). La regulación del Código civil español configura la pena contractual en primer término como sustitutiva de la indemnización de daños y perjuicios prevista en el artículo 1101 del Código civil para el caso de incumplimiento contractual o para los supuestos de cumplimiento deficiente o tardío por parte del deudor. De este modo, se dispensa al acreedor de tener que recurrir a una valoración del daño efectivamente causado, de modo que la cláusula penal viene a ser una estimación anticipada del daño que sustrae al acreedor de la prueba de su existencia. Esta regulación, que se ha mantenido invariable desde la promulgación del Código civil, ha sido objeto de especial atención por parte del Anteproyecto de Modernización del Derecho de Obligaciones elaborado por la Comisión de Codificación del Ministerio de Justicia en 2009, siguiendo las orientaciones presentes en otros Códigos civiles europeos así como las aportaciones de prestigiosos académicos, que son expresión del esfuerzo en pro de la armonización del Derecho contractual europeo.
\end{abstract}


Abstract: The author analyses the legal treatment of the penalty clause into Spanish Civil Law, with special attention to the regulation offered by the Civil Code in their articles 1152 to 1155 , the differents functions which the penalty clause achieves and the differentiated regulation into Fuero Nuevo of Navarra in the Lex 518. All of this can be seen as a reflection of the dualism in Spanish Legal System between the Common Civil Law (represented by the Spanish Civil Code) and the Foral or Special Law (represented by differents regionals compilations). The regulation of the Spanish civil code sets as general rule the contractual penalty as substitute of the regime of compensation into the article 1101 of spanish Civil Code in case of breach of contract or in case of defective or untimely performance by the debtor. This provision allows to the creditor to avoid a real damage assessment, so that the penalty clause lets a advanced estimate of damages without needing to prove them. But also admits the regulation of the penalty clause into the spanish Civil Code other functions, for instance the cumulative penalty (with the legal and previously valued compensation of damages or with the specific performance). This regulation, unchanged since the enactment of the spanish Civil Code, has been object of special attention by the preliminary draft to modernisation of the law of obligations drawn up by the Commission of Codification of the Ministry of Justice in 2009, picking up some of the guidelines present in other European Civil Codes as well as in the contributions of prestigiouses scholars, which are expression of the effort aimed at the harmonisation of European Contract Law.

Palabras clave: cláusula penal, estimación del daño, incumplimiento del contrato, cumplimiento del contrato, armonización del derecho contractual europeo.

Keywords: penalty clause, estimate of damages, breach of contract, performance of contract, harmonisation of European Contract Law.

Recepción original: 17/10/2012

Aceptación original: 24/10/2012

Pretendo ocuparme en este trabajo de algunos de los problemas que surgen del tratamiento que el derecho civil español dedica a la cláusula penal que los contratantes pueden haber convenido para reforzar el cumplimiento de una obligación, llamada por ello principal.

Me referiré también a la peculiaridad de nuestro sistema jurídico, del que resulta que en esta materia la regulación del Código civil no sea aplicable en toda España, significando, en particular, el tratamiento que la Comunidad Foral de Navarra dispensa a la cláusula 
penal, siendo por el momento la única entre la variedad de ordenamientos de derecho civil foral o especial que tenemos en España. Adicionalmente, pondré de relieve cómo detrás de esta diversidad dentro del derecho privado español puede reconocerse un distinto influjo de la experiencia histórica del Derecho romano. Y, por último, en la medida en que esta disciplina (la de la cláusula penal y la de las penas convencionales) ha de enmarcarse dentro del conjunto de garantías que sirven para asegurar el cumplimiento de las obligaciones, parece inevitable perseguir al máximo la armonización en el ámbito espacial de la Unión europea, pues es precisamente el campo de las relaciones jurídico-obligatorios el que se ha mostrado por el momento más propicio para alcanzar la pretendida aproximación entre los distintos ordenamientos jurídicos. Precisamente por ello, las particularidades que ofrece nuestra regulación y que la separa de la que ofrecen otros sistemas de nuestro entorno han sido precisamente objeto de reflexión doctrinal y jurisprudencial. Y ello se ha plasmado, sin que todavía haya visto la luz, en un Anteproyecto que persigue la modernización del libro Cuarto del Código civil en materia de obligaciones y contratos.

El Código civil español dedica a la cláusula penal únicamente cuatro artículos (los artículos 1152 a 1155). En ellos no se recoge un concepto de cláusula penal, de modo que tal tarea ha sido asumida por la doctrina y la jurisprudencia del Tribunal Supremo. En todo caso, de la terminología empleada se desprenden tres elementos que es preciso distinguir con claridad: la cláusula penal, primer elemento, es una convención, autónoma o incorporada a otro negocio, en la que se promete una pena, que sería el segundo elemento, para el caso de incumplimiento, cumplimiento defectuoso o retraso en el cumplimiento de una obligación, que, por ello, podemos llamar «obligación con cláusula penal», erigiéndose así en el tercer elemento.

La cláusula penal cumple variadas funciones según el momento por el que atraviese la obligación principal. Antes de ser exigible el cumplimiento, la función es esencialmente de garantía, reforzando la obligación principal y estimulando al deudor a su cumplimiento ante el eventual endurecimiento de su responsabilidad frente al acreedor. Llegado el vencimiento, la cláusula penal inserta en la obligación principal deviene eficaz y la pena que encierra resulta plenamente exigible cuando se produce el evento concreto para el que estaba prevista. Es la hora de la verdad, el momento en el que la función de garantía o de aseguramiento del crédito da paso a una función de carácter sancionador. 
Esta función punitiva puede tener distinto alcance desde el punto de vista de su intensidad en función de lo convenido por las partes. Si nada se ha pactado, "la pena sustituirá a la indemnización de daños y al abono de intereses en caso de falta de cumplimiento", siempre que la pena «fuere exigible conforme a las disposiciones de este Código» ${ }^{1}$.

En consecuencia, la previsión legal es que la función de la cláusula penal es liquidatoria de la indemnización de daños y perjuicios, pero cabe que los contratantes puedan darle una función cumulativa o, incluso, alternativa con dicha indemnización o con el cumplimiento forzoso.

Es evidente que la pena cumulativa con el cumplimiento forzoso o con la indemnización de daños y perjuicios resulta mucho más coercitiva para el deudor. El derecho del acreedor quedará incólume respecto de la obligación asegurada, pudiendo interesar su cumplimiento en forma específica o, no siendo posible, la indemnización de daños y perjuicios que resultaría de la aplicación del régimen ordinario. Pero además podrá exigir la satisfacción de la pena convenida. De este modo, la función punitiva salta a primera vista.

Con todo, su carácter excepcional, que exige pacto manifiesto, se infiere también del párrafo segundo del art. 1153 del Código civil, que establece que «el acreedor no podrá exigir conjuntamente el cumplimiento de la obligación y la satisfacción de la pena, sin que esta facultad le haya sido claramente otorgada».

Pero también puede hablarse de una función punitiva, un tanto más atenuada, cuando la cláusula penal adopta una función estrictamente liquidadora, sustitutiva de la indemnización de daños y perjuicios. En primer lugar, porque la pena, al ser una estimación anticipada de los daños, puede exceder de los efectivamente ocasionados por el deudor, con lo que, en estos casos, también se daría una ventaja patrimonial para el acreedor.

En segundo lugar, porque la pena es exigible cuando se produce el evento al que se supeditaba. Si la pena había sido prevista para los casos de incumplimiento total, cumplimiento defectuoso o retraso en el cumplimiento, llegada esta circunstancia, la pena es exigible en lugar de la indemnización de daños y perjuicios, sin necesidad de probar la existencia de los mismos ni tampoco su alcance o relación de causalidad con el proceder del deudor. Naturalmente se da la excepción de que el deudor quede liberado en los casos en los que el

${ }^{1}$ Art. 1152 del Código civil. 
incumplimiento tenga lugar por causa ajena a él, con lo que salta así a la vista el carácter accesorio de la cláusula penal, pues no siendo imputable al deudor el incumplimiento de la obligación principal, no resulta exigible la pena convenida.

Por tanto, en esta cláusula penal con función liquidatoria, el carácter sancionador se observa sobre todo en el terreno procedimental, pues el acreedor queda liberado de tener que pasar por las dificultades que siempre concurren en orden a la prueba de los daños y perjuicios efectivamente ocasionados por el incumplimiento del deudor. $\mathrm{O}$, lo que es lo mismo, el hecho de que los contratantes hayan incorporado a la obligación principal una cláusula penal, entraña para el deudor una renuncia a discutir en el proceso el alcance, el importe y relación de causalidad de los daños y perjuicios que invoca el acreedor, puesto que, aunque no se hayan producido, debe satisfacerlos.

Sintetizado así el régimen de la cláusula penal en el Código civil español, se han de significar las particularidades del Derecho navarro. En él, la pena no viene configurada como una liquidación anticipada de los daños y perjuicios, sino como una sanción contra el deudor que incumple la obligación principal. El acreedor, por tanto, además de la acción para exigir el pago de la pena, conserva sus acciones para exigir el cumplimiento forzoso o la indemnización de daños y perjuicios. Cabe incluso pedir daños y perjuicios después de haber cobrado la pena, pero no porque tenga carácter cumulativo, sino porque la pena no ha sido capaz de absorber todos los perjuicios experimentados por el acreedor.

El problema que surge en el régimen del Código civil es cuál es el alcance que ha de darse al futuro 'sustituirá'. ¿Nos encontramos delante de una regla imperativa de modo que una vez incorporada la cláusula penal al negocio principal ello impide al acreedor reclamar la indemnización de daños y perjuicios que resultaría de la aplicación del régimen ordinario, como permite el Derecho navarro?

Cierto es que lo habitual es que el tanto alzado al que se eleva la pena convencional alcance unas proporciones que previsiblemente excedan con mucho de los daños y perjuicios efectivamente ocasionados. Pero cabe considerar la posibilidad de que la estimación anticipada de los daños y perjuicios concretada en la cláusula penal sea ligera o notablemente inferior a los efectivamente producidos por el incumplimiento.

De este modo, el efecto que se produciría sería justo el contrario al que se perseguía, dando así ocasión a que el deudor valore 
en cada caso qué es lo que más le conviene desde un punto de vista patrimonial, esto es, dejar de atender la obligación principal, desencadenando así los efectos de la cláusula penal; o cumplirla puntual y exactamente para evitarla a toda costa.

Y esta preocupación por el carácter imperativo de la regla del art. 1152 del Código civil es todavía mayor si consideramos también otro aspecto crucial tanto en el Código civil como en el Derecho navarro: la moderación judicial de la pena convencional.

Mientras que el art. 1154 del Código civil establece que «el juez modificará equitativamente la pena cuando la obligación hubiera sido en parte o irregularmente cumplida por el deudor», el Fuero Nuevo de Navarra señala que «la pena convenida no podrá ser reducida por el arbitrio judicial» ${ }^{2}$.

El término «moderación» ha de entenderse en el sentido de «minoración» o «disminución» de la pena convenida, de modo que no cabría su «incremento»o «aumento» en aquellos casos en los que la pena convenida, por cualquier circunstancia, se hubiera convertido en irrisoria.

En estos casos, es evidente que el acreedor instará el cumplimiento forzoso en forma específica, pero ¿y si éste ya no fuera posible? ¿Habría de pasar forzosamente el acreedor por la exigencia de la pena convenida o podría acudir a la reclamación de los daños y perjuicios de acuerdo con el régimen legal? Dado el carácter imperativamente sustitutivo, respecto de la indemnización de daños y perjuicios que resulta de la aplicación del régimen ordinario, con el que viene conformada la cláusula penal, parece que el acreedor habría de conformarse con la pena, salvo que otra cosa se hubiese pactado. En cambio, en el Derecho navarro el acreedor podría tranquilamente reclamar la indemnización de daños y perjuicios, renunciando a exigir la pena, pues ésta es siempre subsidiaria, quedando a expensas del acreedor el hacerla valer.

Pero además, el Código civil no admite la moderación en cualquier caso, sino únicamente cuando, estando prevista la pena para un supuesto de incumplimiento total, ha tenido lugar un cumplimiento parcial o defectuoso, con lo que el interés del acreedor no puede considerarse completamente frustrado. No está prevista, a diferencia de los demás ordenamientos jurídicos europeos, la moderación de la pena por excesiva. En cambio, de la contundencia con la

\footnotetext{
${ }^{2}$ Ley 518 del Fuero Nuevo de Navarra.
} 
que viene formulada en el Derecho navarro la prohibición de moderación, ésta no resulta posible en ningún caso.

De todo ello pueden extraerse algunas conclusiones por lo que respecta a la naturaleza jurídica de la cláusula penal. El régimen que nos ofrece el Código civil, que atribuye imperativamente a la cláusula penal el efecto de sustituir a la indemnización de daños y perjuicios que resultaría de la aplicación del régimen ordinario, refleja en primer plano el carácter dispositivo de la cláusula en cuanto expresión de la autonomía de la voluntad de las partes. Si éstas han fijado anticipadamente el importe de los daños y perjuicios, habrán de pasar por él en lo que les favorezca y en lo que les perjudique, no pudiendo nunca hablarse de «pena desorbitada» ni de «pena irrisoria», pues es la que libremente han pactado las partes, renunciando con ello al régimen indemnizatorio ordinario.

Cabe sin embargo, como hemos apuntado, una vía de escape. El art. 1152 no establece imperativamente la sustitución en todo caso. Admite que «otra cosa se pacte». Por tanto, cabe que las partes convengan que la cláusula penal no comporte una renuncia a exigir la indemnización de daños y perjuicios por el cauce ordinario. De este modo, puede decirse que el art. 1153 simplemente ejemplifica los pactos que pueden convenirse: dar a la pena convenida carácter cumulativo $^{3}$ o liberatorio ${ }^{4}$, pero caben otros, como el de facultar al acreedor a reclamar los daños y perjuicios por la vía ordinaria o, simplemente, la cuantía de los mismos no cubierta por la pena.

Sin embargo, el régimen de la moderación de la pena en nuestro Código civil, limitado sólo a los casos de cumplimiento parcial o irregular, es una rara avis. Los ordenamientos jurídicos de nuestro entorno la contemplan, plasmándose de forma explícita en los Códigos civiles de Alemania ${ }^{5}$, Suiza ${ }^{6}$, Austria ${ }^{7}$ e Italia ${ }^{8}$. Incluso, el Code civile

${ }^{3}$ Art. 1153.2: «Tampoco el acreedor podrá exigir conjuntamente el cumplimiento de la obligación y la satisfacción de la pena, sin que esta facultad le haya sido claramente otorgada».

${ }^{4}$ Art. 1153.1: «El deudor no podrá eximirse de cumplir la obligación pagando la pena, sino en el caso de que expresamente le hubiese sido reservado este derecho».

${ }^{5}$ Así el § 343.1 del BGB establece que «ist eine verwirkte Strafe unverhältnismäßig hoch, so kann sie auf Antrag des Schuldners durch Urteil auf den angemessenen Betrag herabgesetzt werden».

${ }^{6}$ El art. 163.3 del Código suizo de Obligaciones señala que las «übermässig hohe Konventionalstrafen hat der Richter nach seinem Ermessen herabzusetzen».

${ }^{7} \mathrm{El} \S 1336.2$ del ABGB dispone que «in allen Fällen ist der Vergütungsbetrag, wenn er vom Schuldner als übermäßig erwiesen wird, von dem Richter, allenfalls nach Einvernehmung von Sachverständigen, zu mäßigen».

${ }^{8}$ El Codice civile establece que «la penale può essere diminuita equamente dal 
francés, en su día fuente de inspiración del Código civil español, tras la reforma de 11 de octubre de 1985, ha ido todavía más lejos, permitiendo que la moderación pueda entrañar un incremento de la pena convenida si ésta hubiera devenido irrisoria9.

Y este criterio generalizado en pro de la moderación de las penas excesivas se encuentra presente en los distintos trabajos que persiguen la armonización del derecho contractual europeo. Así, se contempla en los Principios del Derecho Europeo de Contratos ${ }^{10}$; en el Código Europeo de Contratos a cargo de la Academia de iusprivatistas de Pavía bajo la coordinación del Profesor Giuseppe Gandolfi ${ }^{11}$; también en los Principios UNIDROIT de los contratos comerciales internacionales ${ }^{12}$; y en las Model Rules del Draft Common Frame of Reference ${ }^{13}$.

Esta coincidencia tan generalizada no podía pasar por alto en la nueva regulación de la cláusula penal en la Propuesta de Modernización del Código Civil en materia de Obligaciones y Contratos elaborada por la Sección de Derecho Civil de la Comisión General de Codificación del Ministerio de Justicia de España en 2009 con el propósito de llevar a efecto una reforma de conjunto del Libro Cuarto del Código civil. Así, en el art. 1150 de la Propuesta se establece que «el Juez modificará equitativamente las penas convencionales

giudice, se l'obbligazione principale è stata eseguita in parte ovvero se l'ammontare della penale è manifestamente eccessivo, avuto sempre riguardo all'interesse che il creditore aveva all'adempimento».

${ }^{9}$ Art. 1152: «... néanmoins, le juge peut, même d'office, modérer ou augmenter la peine qui avait été convenue, si elle est manifestement excessive ou dérisoire. Toute stipulation contraire sera réputée non écrite».

$10 \mathrm{El}$ art. 9.509(2) de la versión española dispone que «sin embargo y aun cuando se haya dispuesto otra cosa, la cantidad pactada podrá reducirse a una cifra más razonable, si su importe resultara manifiestamente excesivo en proporción al daño provocado por el incumplimiento y a las demás circunstancias».

${ }^{11} \mathrm{El}$ art. 170.4 señala en su versión española que «La pena puede ser disminuida equitativamente por el juez, cuando el deudor ha efectuado, y si el acreedor no lo ha rechazado, un cumplimiento parcial, o si el montante de la pena es manifiestamente excesivo, habida cuenta, en todo caso, el interés que tenía el acreedor en el cumplimiento».

$12 \mathrm{El}$ art. 7.4.13(2) de los Principios UNIDROIT de los Contratos comerciales internacionales de 2010 establece que «however, notwithstanding any agreement to the contrary the specified sum may be reduced to a reasonable amount where it is grossly excessive in relation to the harm resulting from the non-performance and to the other circumstances".

${ }^{13} \mathrm{El}$ art. III.- 3:712(2) señala que «however, despite any provision to the contrary, the sum so specified in a contract or other juridical act may be reduced to a reasonable amount where it is grossly excessive in relation to the loss resulting from the non-performance and the other circumstances». 
manifiestamente excesivas y las indemnizaciones desproporcionadas en relación con el daño efectivamente sufrido».

Para explicar la diferencia entre el régimen jurídico vigente del Código civil español y del Fuero Nuevo de Navarra es imprescindible considerar la naturaleza jurídica de la stipulatio poenae romana. Como hemos ya apuntado, las diferencias estriban en la ausencia (en el Derecho navarro) de moderación judicial, sea por excesiva que en los supuestos de cumplimiento parcial, y en la exigibilidad de la pena, aunque el incumplimiento sea inimputable al deudor.

El derecho romano no contemplaba la moderación judicial de la pena. Ésta nace de una estipulación, lo que da lugar a un iudicium strictum. Sólo a partir del siglo II d. C. se constata en las fuentes la prohibición de una pena que supere el límite máximo de las usurae centesimae ${ }^{14}$.

Por lo que respecta a la indivisibilidad de la pena en los casos de incumplimiento parcial, tal como se contempla en el Fuero Nuevo de Navarra, el apoyo textual se encuentra en distintos pasajes del Digesto ${ }^{15}$.

También son vacilantes los textos que relacionan la exigencia de la pena con el comportamiento del deudor en relación con la obligación principal. En algunos pasajes ${ }^{16}$ no se exonera de la pena convenida a quien incumple la obligación de entrega de un esclavo por haber éste fallecido o haber sido hurtado, asistiendo así a un supuesto de responsabilidad objetiva por el mero hecho de haberse producido el incumplimiento que condicionaba la exigibilidad de la stipulatio poenae. En cambio, en otro fragmento ${ }^{17}$ en el que las partes, recíprocamente, se habían cruzado distintas stipulationes poenae para el caso de desahucio anticipado por el arrendado y para el supuesto de abandono de la vivienda arrendada antes del plazo convenido, se exime de la obligación de pagar la pena al arrendador por haber desahuciado al arrendatario antes del término fijado por haber incurrido este último en falta de pago de la merced arrendaticia. El fragmento es expresión de la interactuación entre el ius civile y el ius honorarium, corrigiendo este por razones de equidad la rigidez dimante del derecho civil, en tal supuesto mediante una exceptio doli frente a la acción estipulatoria intentada por el arrenda-

${ }^{14}$ Por ejemplo Pap. 11 resp. D. 22.1 .9 pr.; Ulp. 32 ad ed. D. 19.1.13.26; y Mod. 10 pandect. D. 22.1.44.

${ }^{15}$ Entre otros en Ulp. 77 ad ed. D. 2.11.9.1; Pap. 6 resp. D. 19.1.47; y Paul. 75 ad ed. D. 45.1.85.6.

${ }^{16}$ Paul. 22 ad ed. D. 9.2.22 pr. y Cels. 12 dig. D. 47.2.68.1.

${ }^{17}$ Paul. 5 resp. D. 19.2.54.1. 
tario. En todo caso, como Knütel ha manifestado ${ }^{18}$, estos testimonios textuales mostrarían casos singulares producto de la autonomía de las contratantes a la hora de condicionar la exigencia de la pena a la imputabilidad o no del comportamiento del deudor, sin perjuicio de que la naturaleza ex fide bona de las acciones que tutelan la relación arrendaticia haya contribuido a mitigar el rigor de la stipulatio poenae hasta el punto de que no cabía la acumulación de acciones (la del negocio principal y la de la estipulación accesoria) cuando se estaba en presencia de negocios de buena $\mathrm{fe}^{19}$.

Por lo que respecta a la función que cumple la cláusula penal, también la autonomía de la voluntad de los contratantes es decisiva. En algunos casos nos encontramos con un concurso cumulativo de acciones ${ }^{20}$, en otros con un concurso alternativo ${ }^{21}$; finalmente, en otros incluso con la novación de la obligación principal por parte de la cláusula penal ${ }^{22}$.

En la concepción del Código civil, la cláusula penal emerge en primer término como un acuerdo libremente alcanzado por las partes, sustituyendo (si no se ha pactado otra cosa) a la indemnización de daños y perjuicios para el caso de incumplimiento o para el caso de cumplimiento irregular. Siendo la cláusula penal expresión de una lex privata, habrá de pasarse imperativamente por ella, sin permitir más moderación que la que pudiera corresponder a un cumplimiento parcial o irregular, salvo que la pena estuviera prevista precisamente para estos supuestos.

En el Fuero Nuevo de Navarra, con mayor proximidad a las fuentes romanas, se destaca más intensivamente, por el contrario, la función que cumple la cláusula penal en el campo de las relaciones obligatorias, que no es otra que la de estimular a toda costa el cumplimiento de la obligación principal. Una vez establecida la pena y producido el incumplimiento, la pena resulta debida, aunque pueda considerarse desproporcionada y aunque el incumplimiento no sea imputable al deudor.

De este modo, si la cláusula penal siempre favorece el interés del acreedor, en el Fuero de Navarra ello alcanza su máxima expresión, pues, al no constituir una liquidación anticipada de daños y perjui-

${ }^{18}$ R. KNÜTEL, Stipulatio poenae, Köln-Wien 1976, 251 ss.

19 A. FERnández de Buján, Derecho privado romano ${ }^{5}$, Madrid 2012, 593.

${ }^{20}$ Por ejemplo en Pap. 2 quaest. D. 45.1.115.2, donde se concibe el concurso cumulativo como excepcional.

${ }^{21}$ Por ejemplo en Ulp. 20 ad edict. D. 17.2.41 y Iul. 3 ad Urs. Feroc. D. 19.1.28.

${ }^{22}$ Paul. 74 ad ed. D. 44.7.44.6. 
cios, no pierde el acreedor la posibilidad de reclamar la indemnización de daños y perjuicios por el cauce ordinario, a diferencia de lo que sucede en el régimen del Código civil, donde ello sólo será posible si tal posibilidad se ha convenido de forma manifiesta.

A algunos de los problemas a los que da lugar el régimen de la cláusula penal en el Código civil, trata de poner solución la Propuesta de Modernización del Código Civil en materia de Obligaciones y Contratos. Ya nos hemos referido a la moderación judicial de las penas desorbitadas como uno de los aspectos más novedosos de la Propuesta.

Según el art. 1150, la moderación puede actuar desde la equidad frente a las penas excesivas y las indemnizaciones desproporcionadas en relación con el daño efectivamente sufrido, reiterándose aquí la distinción establecida en la Propuesta entre "pena convencional» $\mathrm{e}$ «indemnización previamente establecida» ${ }^{23}$.

Cuando la cláusula cumple una función liquidatoria, ha de guardar relación con el daño efectivamente padecido por el acreedor, lo que parece propugnar por una cláusula penal que ofrezca al acreedor antes ventajas procedimentales para obtener la reparación de su interés patrimonial que las que tendrían un alcance estrictamente cuantitativo. Aún serían éstas posibles, siempre que el montante anticipadamente previsto no tenga el aire de algo desorbitado, pues resulta evidente que no puede pretenderse una coincidencia absoluta si la finalidad de la cláusula penal es ante todo la de evitar las complicaciones que resultarían de su precisa indagación en el curso de un proceso.

En cambio, cuando la pena convenida reviste una función punitiva, la moderación se halla condicionada a que a todas luces sea excesiva. En ello se adivina el influjo de los mencionados trabajos doctrinales en el contexto de la armonización del derecho contractual europeo.

En todo caso, esta moderación ha de respetar el carácter que de forma principal han querido dar los contratantes a la cláusula penal, de modo que en aquellos supuestos en los que se establece acumuladamente con la indemnización que resulta del régimen ordinario ha de primar la consideración de su carácter punitivo, pues la voluntad de las partes ha sido precisamente la de instrumentar

${ }^{23}$ El artículo 1150 de la Propuesta señala que «el Juez modificará equitativamente las penas convencionales manifiestamente excesivas y las indemnizaciones convenidas notoriamente desproporcionadas en relación con el daño efectivamente sufrido». 
una auténtica sanción ante el incumplimiento. Por ello, la minoración que resulte de la moderación no ha de ser de tal intensidad como para desnaturalizar el carácter y función que dimana de la autonomía de los contratantes.

Mayor alcance puede tener la moderación cuando la cláusula penal persigue sustituir a la indemnización, de modo que el carácter punitivo habría de limitarse a las ventajas procedimentales que entraña la adición al negocio principal de una cláusula penal. La renuncia que ello supone para el deudor en orden a la prueba de los perjuicios, su alcance y relación de causalidad con el daño efectuado así como su previsibilidad al tiempo de la celebración del contrato, ya tiene mucho de conminatorio hacia el exacto cumplimiento de lo acordado, pero, en el terreno cuantitativo, el margen con el que cuentan los tribunales para reducir el montante de la pena convenida es ciertamente mucho mayor y habrá de ceñirse en mayor medida al perjuicio ocasionado atendidas todas las circunstancias concurrentes.

Otra de las novedades se halla en el art. 1147 a propósito de la posibilidad de reclamar el exceso cuando la pena convenida, ya percibida, sea insuficiente para cubrir los daños y perjuicios ocasiona$\operatorname{dos}^{24}$. La regla general, sin embargo, es que no cabe tal reclamación, pero se admite por pacto que ello sea posible, de igual modo que en el Código Europeo de Contratos ${ }^{25}$.

El art. 1148 distingue un doble presupuesto para la exigibilidad de la pena. Cuando revista carácter penal, se requiere culpa del deudor. Cuando adopte una función liquidatoria de los daños y perjuicios, basta que el incumplimiento o el cumplimiento defectuoso o retardado, sea imputable al deudor ${ }^{26}$. Se contempla así la distinción entre «incumplimiento imputable al deudor»e «incumplimiento culpable»,

${ }^{24} \mathrm{El}$ art. 1147 de la Propuesta dice concretamente: «la fijación convencional de la indemnización impide al acreedor exigir una cantidad mayor por el daño excedente, salvo que otro hubiera sido el pacto de las partes».

${ }^{25}$ El Artículo 170.1 del Código europeo de Contratos dice que «salvo lo que prevé el párrafo 5, cuando las partes, con motivo de la estipulación del contrato, han convenido en una cláusula penal que, en caso de incumplimiento, de cumplimiento inexacto, o de retraso, sea debida por el deudor una prestación determinada, ésta constituye la reparación que es debida por el deudor, cuando se producen las susodichas situaciones, siempre, salvo que, la reparabilidad del daño ulterior no haya sido convenida».

${ }^{26} \mathrm{El}$ art. 1148 de la Propuesta señala que «el acreedor solo podrá exigir la indemnización previamente convenida cuando el incumplimiento o el cumplimiento defectuoso o retardado sea imputable al deudor. La aplicación de las penas convencionales requerirá la culpa del deudor». 
que estarán en relación de género a especie. Por tanto, tendrá lugar la exoneración de responsabilidad del deudor sólo cuando la imposibilidad de cumplimiento quede absolutamente fuera del círculo de control del deudor, de modo que él no habría podido razonablemente haber previsto ni evitado tales daños y perjuicios.

Para concluir, el art. 1149 pone de manifiesto expresamente la incompatibilidad entre el ejercicio de la acción de cumplimiento en forma específica y la ordenada a exigir la pena convenida, salvo que el cumplimiento deviniera imposible o que la pena sancione el retraso en el cumplimiento ${ }^{27}$.

Por consiguiente, a diferencia de nuestro actual Código civil, que permite en su art. 1153 el pacto de reclamar el cumplimiento forzoso de la obligación principal junto con la pena convenida, cuando la cláusula penal adopta carácter punitivo, resulta posible la reclamación conjunta de la reparación ordinaria de los daños y perjuicios, conforme al art. 1101 del Código civil, y la pena convenida. Por el contrario, fuera de las excepciones referidas, no puede el acreedor entablar exitosamente ambas acciones.

\section{BIBLIOGRAFÍA}

M. ALBALADEJO, Artículos 1152 y 1153, en M. ALBALADEJO (Dir), Comentarios al Código civil y compilaciones forales, 15.2, Madrid 1986.

M. T. ALONSO PÉREZ, Remedios, en G. GARCÍA CANTERO (Coord.), Anotaciones españolas al Proyecto de Pavía, Zaragoza 2005, 797-862.

I. ARANA DE LA FUENTE, Algunas precisiones sobre la reforma de la cláusula penal en la Propuesta de Modernización del Código Civil en materia de Obligaciones y Contratos, en InDret 4 (2010), 3-19.

P. BENJAMíN, Liquidated Damages and Penal Clauses in Commercial Contracts: a Comparative Study of English and Continental Law, en The International and Comparative Law Quaterly 9.4 (1960) 600-627.

${ }^{27}$ Así, el artículo 1149 reza del siguiente modo: «el ejercicio de la acción de cumplimiento en forma específica impide al acreedor reclamar la indemnización convenida de los daños y la pena convencional, salvo que éstas hubiesen sido estipuladas para el caso de retraso o que el cumplimiento en forma específica resulte imposible. Si el acreedor obtiene la resolución por incumplimiento, tendrá derecho a las indemnizaciones para el supuesto de aquella pactadas y a las penas convencionales pactadas para el cumplimiento retrasado». 
J. BIANCALANA, The Development of the Penal Bond with Conditional Defeasance, en Journal of Legal History 26.2 (2005) 103-117.

J. J. BLANCO GÓMEZ, La cláusula penal en las obligaciones civiles: relación entre la prestación penal, la prestación principal y el resarcimiento del daño, Madrid 1996.

X. O'CALlaghaN, Compendio de derecho civil, 2, Obligaciones y contratos, 1, Madrid 2002.

J. DÁVILA GONZÁLEZ, La obligación con cláusula penal, Madrid 1992.

G. DE CASTRO VÍTORES, La cláusula penal ante la armonización del derecho contractual europeo, Madrid 2009.

I. ESPÍN ALBA, La cláusula penal. Especial referencia a la moderación de la pena, Madrid 1997.

D. ESPÍN CÁNOVAS, La cláusula penal en las obligaciones contractuales, en Revista de Derecho Privado 30.348 (1946) 145-169.

A. FERNÁNDEZ DE BUJÁN, Derecho privado romano ${ }^{5}$, Madrid 2012.

C. FUENTESECA DEGENEFFE, Diversos matices de la regulación de la cláusula penal en el ordenamiento español y alemán, en Foro 13 (2011), 61-110.

C. J. GOETZ - R. E. SCOTT, Liquidates Damages, Penalties and the just Compensation Principle: some Notes on an Enforcement Model and a Theory of efficient Breach, en Columbia Law Review 77.4 (1977) 554-594.

R. KNÜTEL, Stipulatio poenae. Studien zur römischen Vertragstrafe, Köln-Wien 1976.

J. M. LOBATO DE BLAS, La cláusula penal en el derecho español, Pamplona 1974.

J. M. MANRESA, Comentarios al Código civil español², 8, Madrid 1907.

I. MARÍN GARCÍA, La cláusula penal en la Propuesta de Anteproyecto de Ley de Modernización del Derecho de Obligaciones y Contratos, en InDret 2 (2009) 2-17.

F. MARTÍNEZ MAS, La cláusula penal en el contrato de obra, Las Palmas de Gran Canaria 2005.

U. MATTEI, The Comparative Law and Economics of Penalty Clauses in Contracts, en The American Journal of Comparative Law 43.3 (1995) 427-444. 
V. MONTÉS PENADÉS, Las garantías del crédito, en M. ${ }^{\mathrm{a}} \mathrm{R}$. VALPUESTA (Coord.), Derecho de obligaciones y contratos ${ }^{2}$, Valencia 1995.

S. NAVAS NAVARRO, El incumplimiento no esencial de la obligación. Análisis del incumplimiento no esencial de las obligaciones contractuales de dar, Madrid 2004.

R. M. ROCA SASTRE - J. PUIG BRUTAU, La cláusula penal en las obligaciones contractuales, en Estudios de Derecho Privado, Tomo I, Volumen VIII, Obligaciones y Contratos, Madrid 1948, 269-285.

J. M. RODRÍGUEZ TAPIA, Sobre la cláusula penal en el Código civil, en Anuario de Derecho Civil 46.2 (1993), 511-587.

E. RUIZ VADILLO, Algunas consideraciones sobre la cláusula penal, en Revista de Derecho Privado 59 (1975), 374-412.

W. SACHER, Vertragsstrafe und Schadenspauschalierung im spanischen Recht. Die cláusula penal und ihre Berücksichtigung im rahmen des deutschen AGB-Gesetzes, München 1981.

A. M. SANZ VIOLA, La cláusula penal en el Código civil, Barcelona 1994.

T. SEGRÈ, Clause pénale et dommages ultérieurs en droit comparé, en Revue Internationale de droit comparé 22.2 (1979), 299-311.

A. SICARI, Pena convenzionale e responsabilità, Bari 2001.

G. SMORTO, I criteri di determinazione del danno nelle model rules e nel diritto commune europeo, en Europa e Diritto privato 1 (2011).

L. A. SOLER PASCUAL, Los consumidores ante la adquisición de un bien inmueble en proyecto o en fase de construcción, en Revista de la Facultad de Ciencias Sociales y Jurídicas de Elche 1.3 (2008), 93-106.

M. TALAMANCA, s.v. pena privata (diritto romano), en Enciclopedia del Diritto 32 (1982).

M. UREÑA MARTÍNEZ, La cláusula penal en el contrato de leasing, Madrid 2003.

R. ZIMMERMANN, Ius commune and the Principles of European Contract Law: Contemporary Renewal of an Old Idea, en H. L. MCQUEEN y R. ZIMMERMANN (Eds.), European Contract Law: Scots and South African Perspectives, Edinburgh 2006, 1-42. 